\title{
Misdiagnosis of Mullerian agenesis in a patient with 46, XX gonadal dysgenesis: a missed opportunity for prevention of osteoporosis
}

\author{
Yotsapon Thewjitcharoen, Veekij Veerasomboonsin, Soontaree Nakasatien, \\ Sirinate Krittiyawong and Thep Himathongkam
}

Diabetes and Thyroid Center, Theptarin Hospital, Bangkok, Thailand
Correspondence should be addressed to $Y$ Thewjitcharoen Email

kamijoa@hotmail.com

\section{Summary}

Primary amenorrhea could be caused by disorders of four parts: disorders of the outflow tract, disorders of the ovary, disorders of the anterior pituitary, and disorders of hypothalamus. Delay in diagnosis and hormone substitution therapy causes secondary osteoporosis. Herein, we report a case of a 23-year-old phenotypical female who presented with primary amenorrhea from 46, XX gonadal dysgenesis but had been misdiagnosed as Mayer-Rokitansky-Kuster-Hauser (MRKH) syndrome or Mullerian agenesis. The coexistence of gonadal dysgenesis and MRKH was suspected after laboratory and imaging investigations. However, the vanishing uterus reappeared after 18 months of hormone replacement therapy. Therefore, hormone profiles and karyotype should be thoroughly investigated to distinguish MRKH syndrome from other disorders of sex development (DSD). Double diagnosis of DSD is extremely rare and periodic evaluation should be reassessed. This case highlights the presence of estrogen deficiency state, the uterus may remain invisible until adequate exposure to exogenous estrogen.

\section{Learning points:}

- An early diagnosis of disorders of sex development (DSD) is extremely important in order to promptly begin treatment, provide emotional support to the patient and reduce the risks of associated complications.

- Hormone profiles and karyotype should be investigated in all cases of the presumptive diagnosis of MayerRokitansky-Kuster-Hauser (MRKH) syndrome or Mullerian agenesis.

- The association between $46, X X$ gonadal dysgenesis and Mullerian agenesis has been occasionally reported as a co-incidental event; however, reassessment of the presence of uterus should be done again after administration of exogenous estrogen replacement for at least 6-12 months.

- A multidisciplinary approach is necessary for patients presenting with DSD to ensure appropriate treatments and follow-up across the lifespan of individuals with DSD.

\section{Background}

Disorders of sex development (DSD) have genetically and clinically heterogeneous phenotypes and are rarely seen in their clinical practice (1). DSD can present to various medical specialists and at different ages. Over the last decade, both genetic diagnosis and clinical treatments have advanced considerably but the fundamental concept 
remains the same in which the endocrinologist plays a key role in coordinating investigations and management in the context of multidisciplinary approach.

In patients who presented with primary amenorrhea, Mayer-Rokitansky-Kuster-Hauser (MRKH) syndrome or Mullerian agenesis is one of the differential diagnosis in patients with 46, XX karyotype. It is reported to be the cause of primary amenorrhea up to 15\% (2). Patients with Mullerian agenesis lack all derivatives of the mullerian ducts (fallopian tubes, uterus, cervix, and upper vagina) but have ovaries and undergo puberty, with appropriately timed breast development and growth of axillary and pubic hair. Hormone profiles and associated congenital anomalies should be thoroughly investigated in all cases of the presumptive diagnosis of this condition. Determining the presence or absence of secondary sexual characteristics especially breast development is a simple first step to ensure the presence of circulating estrogen level (3). In adolescent or adult patients without the secondary sexual characteristics, other differential diagnoses or the probability of co-occurrence of other diseases are warranted (4). It should be emphasized that the uterus may not be visible to various imaging modalities even to laparoscopy in patients with severe estrogen deficiency (5). Therefore, the diagnosis of Mullerian agenesis should be re-evaluated again after at least 6-12 months of exogenous estrogen replacement.

Herein, we describe an interesting case of a female patient who presented with primary amenorrhea since the age of 16 years and had been misdiagnosed as Mullerian agenesis without additional hormonal tests. This case highlights the importance of detailed investigations before making a presumptive diagnosis of Mullerian agenesis

\section{Case presentation}

A 23-year-old virgin Thai female presented with primary amenorrhea and poor breast development. Her perinatal and neonatal period was uneventful. The patient had no family history of significant congenital abnormalities. She was evaluated with the problem of primary amenorrhea from a local gynecologist at the age of 16 years. Only karyotype and pelvic ultrasonography were tested with no further evaluations. The chromosome study confirmed normal 46, XX karyotype. No details of pelvic ultrasonography were obtained, but the patient had been told that her uterus was absent. No treatment was given at that time and she was lost to follow-up after a few clinic visits. After having been given diagnosis of MRKH syndrome, she never menstruated and had no breast development.

At the initial visit at our hospital, her height and weight were $157 \mathrm{~cm}$ and $49 \mathrm{~kg}$, respectively. There was no feature suggestive of Turner syndrome and no skeletal deformity was found. The secondary sexual characteristics assessment showed breast Tanner stage I and pubic hair stage III. Female external genitalia and a normal vagina ending in a blind pouch were found at the gynecological exam. The remainder of the physical examination was normal.

\section{Investigation}

Due to the absence of breast development, the presumptive diagnosis of MRKH syndrome or Mullerian agenesis was questioned. The initial laboratory investigation in our hospital revealed hypergonadotrophic hypogonadism (FSH $130 \mathrm{IU} / \mathrm{L}, \mathrm{LH} 2 \mathrm{IU} / \mathrm{L}$, serum estradiol $<5 \mathrm{pg} / \mathrm{mL}$ ) with confirmed 46, XX karyotype. Internal genitalia (ovaries, uterus, and upper two third of vagina) and streak gonad could not be identified on the pelvic magnetic resonance imaging (MRI). Other investigations including complete blood count, renal function, liver function, prolactin hormone, and thyroid function test revealed normal results. Bone density scan (DEXA scan) revealed osteoporosis at the lumbar spine ( $\mathrm{T}$ score -2.7 ) and osteopenia at the hip (T score -1.5). The diagnosis of 46, XX gonadal dysgenesis associated with MRKH syndrome was suspected based on both absence of uterus and ovaries. Skeletal survey by X-rays revealed no associated anomalies of the spines. The DNA sequence analysis included potential genes panel associated with 46, XX gonadal dysgenesis (WNT4, WNT9, RSPO1, SOX9, NROB1, GATA4, STAR, WT1) revealed no pathogenic variants.

\section{Treatment}

The patient was started on hormonal substitution therapy with $0.625 \mathrm{mg}$ of daily oral conjugated equine estrogen (Premarin). Six months later, the patient showed a Tanner breast stage III and further developed into a Tanner breast stage $\mathrm{V}$ at 18 months after treatment. Oral calcium and vitamin $\mathrm{D}$ were also given to prevent further bone loss. The improvement of bone mineral density was observed at the repeated DEXA scan at 18 months but still revealed osteopenia at the lumbar spine ( $\mathrm{T}$ score at the lumbar spine increased from -2.7 to -2.2 and $\mathrm{T}$ score at the hip increased from -1.5 to -1.3 ). 


\section{Outcome and follow-up}

An ultrasound of the pelvis was repeated at 18 months. Unexpectedly, newly detected $1.3 \times 3.8 \mathrm{~cm}$ rudimentary uterine buds were noted with no demonstration of both ovaries and upper part of vagina. Pelvic MRI was done to confirm the presence of a growing uterus at 24 months (uterine dimension $1.8 \times 2.9 \times 4.9 \mathrm{~cm}$, endometrial thickness $1.3 \mathrm{~cm}$ ) after the beginning of estrogen treatment as shown in Fig. 1. Based on the reappearance of a normal uterus which has a size equivalent to a normal 16-year-old girl, the diagnosis of pure 46, XX gonadal dysgenesis only without Mullerian agenesis was established. The patient's treatment had been converted from estrogen only into a cyclical oral estrogen/progesterone replacement therapy (10 mg of cyclic medroxyprogesterone acetate 10 days in each cycle) to prevent unopposed estrogen effect on uterus. At present, no breakthrough menstruation occurs yet. She has been informed about future options for having children by adoption or gestational surrogacy. The patient was able to cope with her definite diagnosis and understand her disease very well.

\section{Discussion}

In the present case, we pointed out the critical gaps in misdiagnosis of MRKH syndrome or Mullerian agenesis in a patient with $46, \mathrm{XX}$ gonadal dysgenesis. Such a diagnosis has therapeutic and psychological consequences. MRKH syndrome is estimated to occur in as many as one in 5000 women and is usually first recognized in late puberty when menstruation fails to occur (2). But it should be emphasized that this condition has normal ovarian function. Hormonal investigations are mandatory to ensure the right diagnosis. Another important clue in this patient is lack of breast development which should signal the possibility of estrogen deficiency. For patients in a prepubertal state, premature diagnosis of $\mathrm{MRKH}$ syndrome should be cautioned from underdevelopment of uterus which could be missed in all imaging modalities from very tiny size of uterine bud. Even laparoscopy can miss a small uterus due to limitation of surgical field.

The issue of uterine hypoplasia in patients with severe estrogen deficiency was reported in 2010 (5), but this potential pitfall in diagnosis has only recently been highlighted in the recent article (6). The dual diagnosis of MRKH syndrome and 46, XX gonadal dysgenesis (absence of uterus and both ovaries) in previous case reports were questioned because most reports did not have follow-up imaging of uterus again after adequate estrogen replacement $(7,8,9)$. Subsequent identification of the uterus needs to be re-evaluated after at least 6-12 months of estrogen replacement. Treatment with estrogen and progesterone are required for reducing the risk of endometrial hyperplasia and carcinoma, which will increase due to long-term application of estrogen without opposition. In our patient, a rudimentary uterus was suspected from pelvic ultrasonography at 18 months
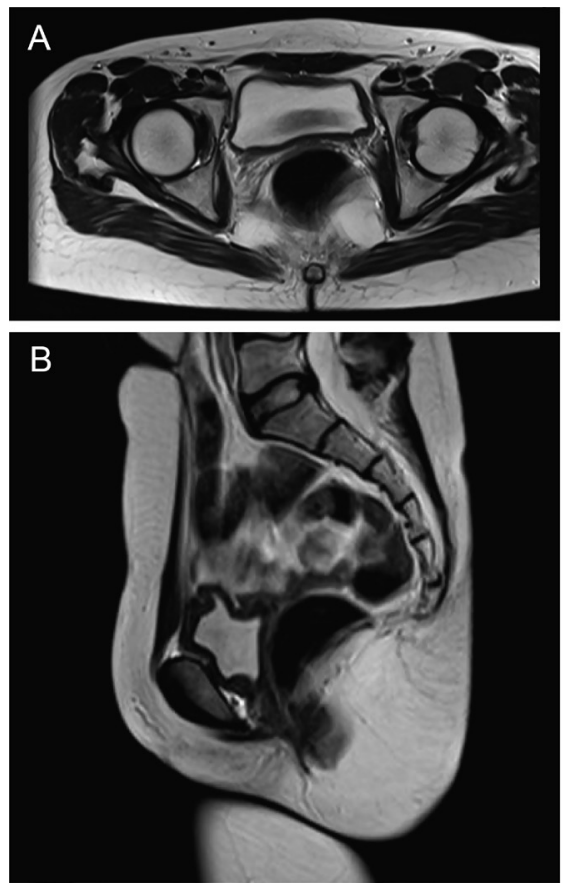
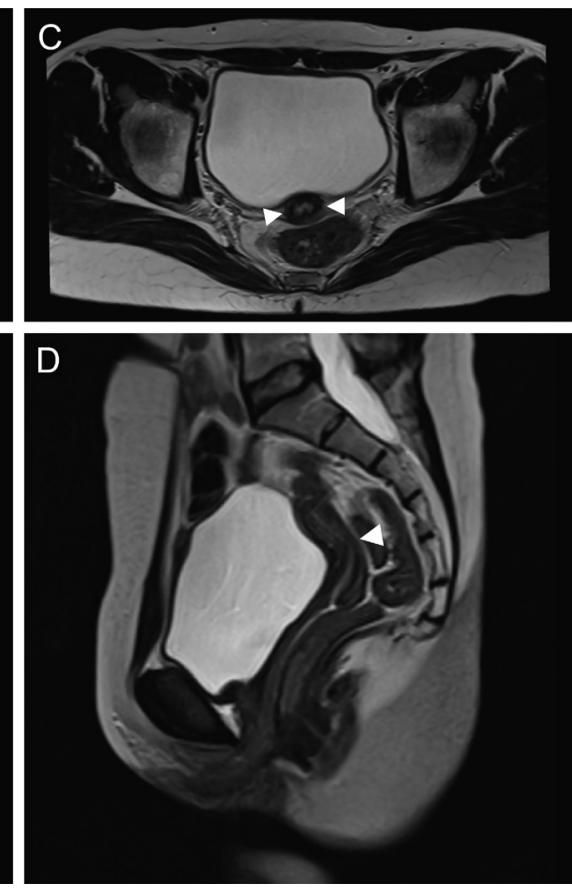

Figure 1

MRI of the pelvis before and after estrogen replacement for 24 months. (A) An axial

T2-weighted image showed bladder and rectum without interposition of uterus before estrogen replacement. (B) A sagittal T2-weighted image revealed the absence of uterus and ovaries before estrogen replacement. (C) An axial T2-weighted image at 24 months after estrogen treatment revealed the presence of growing uterus and upper vagina shows a vagina (arrowheads). (D) A sagittal T2-weighted image after estrogen replacement (arrowheads). 
after the start of estrogen replacement and then later was confirmed by pelvic MRI. Uterus could continue to grow into adult size after several years of estrogen administration and these patients might have successful pregnancy with in vitro fertilization using donor oocytes.

Gonadal dysgenesis is an infrequent cause for primary amenorrhea (less than 1:100 000) which some cases had been associated with insufficient expression of pro-ovarian genes (such as WNT4, RSPO1, FOXL2, etc.) (10). However, many genes for ovarian development remain unknown and in most cases of $46, \mathrm{XX}$ gonadal dysgenesis the cause remained unidentified candidate genes. From a clinical point of view, clinicians should focus on long-term effects of estrogen deficiency which represents an important risk factor for neurological, metabolic, cardiovascular health problems and bone health problems. Early initiation of estrogen therapy is essential to minimize bone loss. Moreover, lifelong oral calcium and vitamin $\mathrm{D}$ supplement should be given to maintain bone health. Bisphosphonates should be used only in patients with severe bone loss and in which estrogen replacement therapy failed to improve the bone condition.

In conclusion, we reported a case of female patient who presented with primary amenorrhea from 46, XX gonadal dysgenesis but had been misdiagnosed as MRKH syndrome. Pelvic ultrasonography only without further investigations can be misleading in the evaluation of primary amenorrhea. In the presence of severe estrogen deficiency, premature diagnosis of MRKH syndrome should be avoided. Focused physical examinations and hormone profiles are necessary to distinguish MRKH syndrome from other disorders of sex development. An early and accurate diagnosis of $46, \mathrm{XX}$ gonadal dysgenesis is important for the provision of proper long-term management and of emotional support to the patient.

\section{Declaration of interest}

The authors declare that there is no conflict of interest that could be perceived as prejudicing the impartiality of this case report.

\section{Funding}

This research did not receive any specific grant from any funding agency in the public, commercial or not-for-profit sector.

\section{Patient consent}

Written informed consent was obtained from the patient for publication of the submitted article.

\section{Author contribution statement}

$Y$ Thewjitcharoen wrote the initial draft of the article, and all authors contributed to the editing process. $Y$ Thewjitcharoen was involved in the direct care of the patient and $\mathrm{V}$ Veerasomboonsin provided expert opinion on all radiological investigations undertaken. Parts of this manuscript had previously been presented as a poster at ENDO 2018, the Endocrine Society's annual meeting 2018, Chicago, USA.

\section{References}

1 Hiort O, Birnbaum W, Marshall L, Wünsch L, Werner R, Schröder T, Döhnert U \& Holterhus PM. Management of disorders of sex development. Nature Reviews: Endocrinology 201410 520-529. (https://doi.org/10.1038/nrendo.2014.108)

2 Oppelt P, Renner SP, Kellermann A, Brucker S, Hauser GA, Ludwig KS, Strissel PL, Strick R, Wallwiener D \& Beckmann MW. Clinical aspects of Mayer-Rokitansky-Kuester-Hauser syndrome: recommendations for clinical diagnosis and staging. Human Reproduction 200621 792-797. (https://doi.org/10.1093/humrep/dei381)

3 Klein DA, Paradise SL \& Reeder RM. Amenorrhea: a systematic approach to diagnosis and management. American Family Physician $201910039-48$.

4 Rall K, Eisenbeis S, Henninger V, Henes M, Wallwiener D, Bonin M \& Brucker S. Typical and atypical associated findings in a group of 346 patients with Mayer Rokitansky-Kuester-Hauser syndrome. Journal of Pediatric and Adolescent Gynecology 201528 362-368. (https://doi. org/10.1016/j.jpag.2014.07.019)

5 Michala L, Aslam N, Conway GS \& Creighton SM. The clandestine uterus: or how the uterus escapes detection prior to puberty. BJOG 2010117 212-215. (https://doi.org/10.1111/j.14710528.2009.02413.x)

6 Berglund A, Burt E, Cameron-Pimblett A, Davies MC \& Conway GS. A critical assessment of case reports describing absent uterus in subjects with oestrogen deficiency. Clinical Endocrinology 201990 822-826. (https://doi.org/10.1111/cen.13963)

7 Bousfiha N, Errarhay S, Saadi H, Ouldim K, Bouchikhi C \& Banani A. Gonadal dysgenesis 46, XX associated with Mayer-Rokitansky-Kuster-Hauser syndrome: one case report. Obstetrics and Gynecology International 20102010 847370. (https:// doi.org/10.1155/2010/847370)

8 Manne S, Veeraabhinav CH, Jetti M, Himabindu Y, Donthu K $\&$ Badireddy M. A rare case of 46, XX gonadal dysgenesis and Mayer-Rokitansky-Kuster-Hauser syndrome. Journal of Human Reproduction Science 20169 263-266. (https://doi.org/10.4103/09741208)

9 Kisu I, Ono A, Iijma T, Katayama M, Iura A \& Hirao N. MayerRokitansky-Küster-Hauser syndrome with a uterine cervix and normal vagina associated with gonadal dysgenesis in a 46,XX female. Journal of Obstetrics and Gynaecology Research 201945 1386-1390. (https://doi.org/10.1111/jog.13956)

10 Ono M \& Harley VR. Disorders of sex development: new genes, new concepts. Nature Reviews: Endocrinology 20139 79-91. (https://doi. org/10.1038/nrendo.2012.235) 